\title{
Tau energy calibration in the ATLAS experiment
}

\author{
Amelia BRENNAN* on behalf of the ATLAS Collaboration \\ The University of Melbourne \\ E-mail: amelia.jean.brennanecern.ch
}

We describe the energy scale calibration of hadronic $\tau$ decays and the associated uncertainty using $4.5 \mathrm{fb}^{-1}$ of data at $\sqrt{s}=8 \mathrm{TeV}$ recorded in 2012 with the ATLAS detector at the LHC. The calibration is based on simulated $\tau$ decays, while the systematic uncertainty includes contributions from the single particle response measurements, pile-up and material modeling. The systematic uncertainty on the hadronic $\tau$ energy scale for $p_{\mathrm{T}}^{\tau}>20 \mathrm{GeV}$ and $\left|\eta^{\tau}\right|<2.5$ is found to be $\leq 3 \%$ for the hadronic decay modes with exactly one reconstructed track, and $\leq 4 \%$ for the hadronic decay modes with at least two reconstructed tracks. The systematic uncertainty is obtained with a deconvolution method, and is checked using an in-situ analysis of the visible mass of reconstructed $Z$ boson decays into one leptonically and one hadronically decaying $\tau$. These two methods yield results that are compatible within the calculated uncertainties.

The European Physical Society Conference on High Energy Physics

18-24 July, 2013

Stockholm, Sweden

${ }^{*}$ Speaker. 
Understanding the decay of tau leptons is important in Large Hadron Collider (LHC) physics; they are relevant for the phenomenology of the Higgs boson, and in searches for physics beyond the Standard Model, including those searches performed at the ATLAS experiment [1]. Tau leptons decay hadronically $65 \%$ of the time, predominantly to one or three charged pions (1-prong and multi-prong taus, respectively), a neutrino, and often additional neutral pions. The energies of these pions are calibrated using the jet energy scale, however this hadronic pre-calibration does not account for energy lost before the calorimeters and other effects, and the average difference between the reconstructed and true hadronic tau $\left(\tau_{\text {had }}\right)$ energies can remain as large as $15 \%$ at low $p_{\mathrm{T}}^{\tau}$ [2]. This indicates the need for an additional correction to the calibration of the visible energy.

The calibrated momentum $p_{\text {cal }}^{\tau}$ is defined as

$$
p_{\mathrm{cal}}^{\tau}=\frac{p_{\mathrm{LC}}^{\tau}}{R\left(p_{\mathrm{LC}}^{\tau}, \mid \eta_{\text {reco }}^{\tau}, n_{p}\right)},
$$

where $p_{\mathrm{LC}}^{\tau}$ is the reconstructed $\tau_{\text {had }}$ momentum at the Local Hadron Calibration (LC) scale, $\eta_{\text {reco }}^{\tau}$ is the reconstructed $\tau_{\text {had }}$ pseudorapidity, $n_{p}$ is the number of reconstructed tracks and $R$ is the additional calibration term, termed the response. $R$ is defined as the ratio of $p_{\mathrm{LC}}^{\tau}$ to the true $\tau_{\text {had-vis }}$ momentum $\left(p_{\mathrm{vis}}^{\tau-\text { true }}\right)$, as a function of $p_{\mathrm{vis}}^{\tau-\text { true }},\left|\eta_{\mathrm{reco}}^{\tau}\right|$ and $n_{p}$. The response curves are shown in Fig. 1 as a function of the reconstructed $\tau_{\text {had }}$ momentum at the LC scale for $\tau_{1-\text { prong }}$ (left) and $\tau_{\text {multi-prong }}$ (right). These response curves represent a measure of the average $p_{\mathrm{T}}^{\text {reco }} / p_{\mathrm{T}}^{\text {truth }}$ before the calibration is applied.
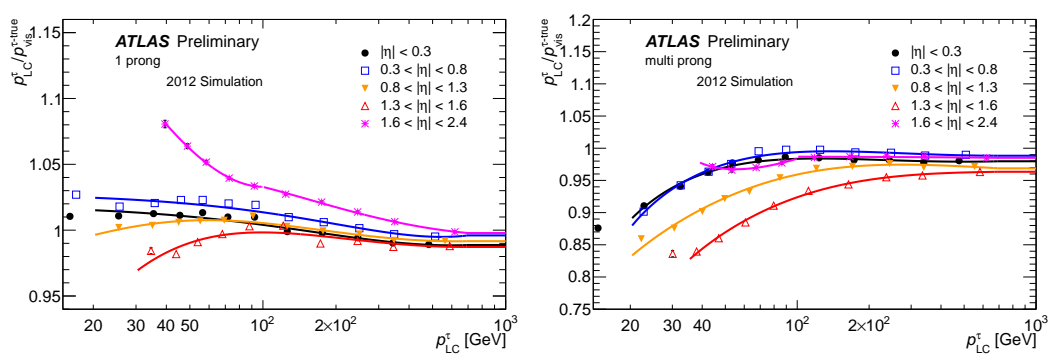

Figure 1: Response curves as a function of the reconstructed $\tau_{\text {had }}$ momentum at LC scale for

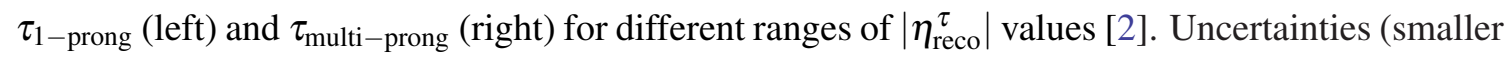
than the shown markers in most bins) are statistical only.

The systematic uncertainties considered are shown in Fig. 2. The single particle response is derived from the calorimeter uncertainty, and includes contributions from the combined testbeam (CTB) data [3], $\langle E / p\rangle$ in-situ measurements [4], and the EM energy response. Together, the systematic uncertainty, across most $\left|\eta_{\tau}\right|$ and $p_{\mathrm{T}}$ bins, is between $2-3 \%\left(\tau_{1-\text { prong }}\right)$ and $2-3.5 \%$ $\left(\tau_{\text {multi-prong }}\right)$ for taus passing the medium identification criteria, and between $2-4 \%$ ( $\left.\tau_{1-\text { prong }}\right)$ and $2.5-4 \%\left(\tau_{\text {multi-prong }}\right)$ for taus passing the tight identification criteria ${ }^{1}[2]$. The maximum uncertainties are on multi-prong taus in the region $1.3<\left|\eta^{\tau}\right|<1.6$, in the lowest $p_{\mathrm{T}}$ bin.

An in-situ cross-check is performed using the visible mass peak of the $Z \rightarrow \tau \tau \rightarrow \mu \tau_{\text {had }}$ final state to measure the TES and associated uncertainty, in particular to verify our procedure in the region $\left|\eta^{\tau}\right|>0.8$ where there is no CTB data available. Small shifts in the $Z$ visible mass are

\footnotetext{
${ }^{1}$ See Ref. [5] for a description of the use of a Boosted Decision Tree algorithm to define these identification criteria.
} 

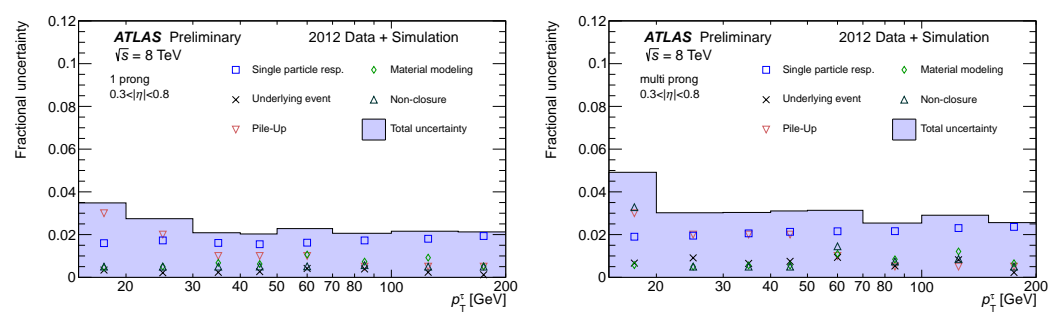

Figure 2: TES uncertainty for $\tau_{1-\text { prong }}$ (left) and $\tau_{\text {multi-prong }}$ (right) for $0.8<\left|\eta^{\tau}\right|<1.3$ [2]. The individual contributions are shown as points and the combined uncertainty is shown as a filled band. Bins in $p_{T}^{\tau}$ with equal uncertainties are grouped.
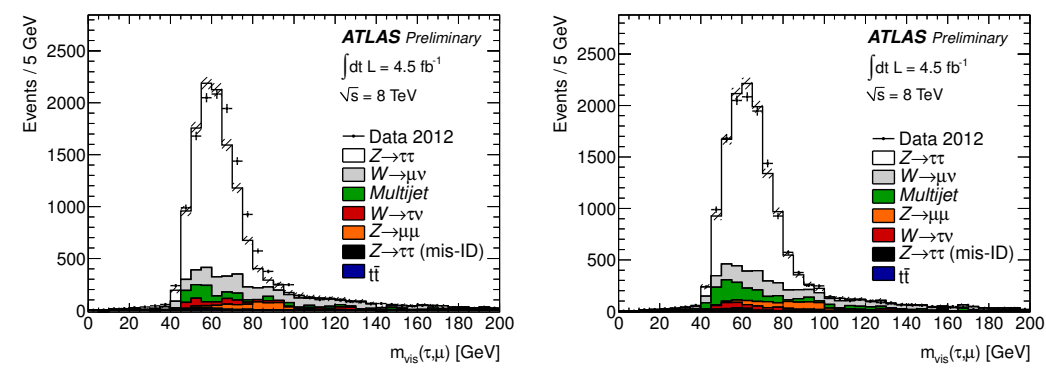

Figure 3: Templates for $0.8<\left|\eta^{\tau}\right|<2.5$ for values of $\alpha$ of $-10 \%$ (left), and the best match with the data (right) [2].

proportional to shifts in the tau transverse momentum, $p_{\mathrm{T}}^{\tau}$, so we can shift $p_{\mathrm{T}}^{\tau}$ in simulation according to $p^{\prime}{ }_{\mathrm{T}}^{\tau}=(1+\alpha) p_{\mathrm{T}}^{\tau}$, and compare the position of the visible mass peak to that in data. Fig. 3 shows the visible mass peak of the $Z$ in the region $0.8<\left|\eta^{\tau}\right|<2.5$ for $\alpha=-10 \%$ (left), and for the optimal value of $\alpha=-1.6 \%$ (right). In the low $\left|\eta^{\tau}\right|$ region, $\alpha$ is calculated to be $-3.0 \%$, and so the difference between the two regions is $(1.4 \pm 3.6) \%$ [2], with the systematic uncertainties calculated by varying each source of uncertainty of uncertainty and recalculating the TES. This is interpreted as indicating no significant difference between the two $\left|\eta^{\tau}\right|$ regions.

\section{References}

[1] ATLAS Collaboration, The ATLAS Experiment at the CERN Large Hadron Collider, JINST 3 (2008) S08003

[2] ATLAS Collaboration, Determination of the tau energy scale and the associated systematic uncertainty in proton-proton collisions at at $\sqrt{s}=8 \mathrm{TeV}$ with the ATLAS detector at the LHC in 2012, ATLAS-CONF-2013-044

[3] E. Khramov et al., Study of the Response of the Hadronic Barrel Calorimeter in the ATLAS Combined Test-beam to Pions of Energies from 20 to $350 \mathrm{GeV}$ for Beam Impact Points from 0.2 to 0.65, ATL-TILECAL-PUB-2009-007

[4] ATLAS Collaboration, ATLAS Calorimeter Response to Single Isolated Hadrons and Estimation of the Calorimeter Jet Scale Uncertainty, ATLAS-CONF-2011-028

[5] ATLAS Collaboration, Identification of the Hadronic Decays of Tau Leptons in 2012 Data, ATLAS-CONF-2013-064 